$\mathrm{BMB}$

BMB Rep. 2017; 50(6): 281-282

Reports

www.bmbreports.org

Perspective

\title{
Deubiquitinase YOD1: the potent activator of YAP in hepatomegaly and liver cancer
}

\author{
Youngeun Kim E Eek-hoon Jho* \\ Department of Life Science, University of Seoul, Seoul 02504, Korea
}

\begin{abstract}
Advances in the understanding of the Hippo signaling as a key regulatory pathway of proliferation and apoptosis have provided mechanical insights for controlling organ size and tumorigenicity. Recently, much attention has been directed to the regulation of LATS1/2 (large tumor suppressor) kinases that phosphorylate YAP/TAZ, a transcriptional co-activator in the Hippo pathway, and control the level and nuclear localization of YAP/TAZ. In our recent work, we showed that deubiquitinase YOD1 stabilizes ITCH, and facilitates ITCH-mediated LATS1/2 ubiquitination and degradation, resulting in increased YAP/TAZ level. Furthermore, we found that the YOD1ITCH-LATS1/2-YAP/TAZ signaling axis is controlled by the differential expression of miR-21 in a cell-density-dependent manner. Using a transgenic mouse model, we showed that the inducible expression of YOD1 enhances the proliferation of hepatocytes and leads to hepatomegaly in a YAP/TAZ-activitydependent manner. Moreover, a strong correlation was observed between YOD1 and YAP expression in liver cancer patients. Overall, our data suggest that YOD1 is a novel regulator of the Hippo pathway, and thereby a potential therapeutic target for liver cancer. [BMB Reports 2017; 50(6): 281-282]
\end{abstract}

The Hippo pathway restricts cell proliferation and plays key roles in controlling organ size and tissue homeostasis. In a high cell densities, the Hippo pathway is initiated by activating

*Corresponding author. E-mail: ej70@uos.ac.kr

https://doi.org/10.5483/BMBRep.2017.50.6.078

Received 13 May 2017

Keywords: Deubiquitinase, Hippo signaling, ITCH, microRNA, YOD1

Abbreviations: Dox, Doxycycline; DUB, Deubiquitinase; ER, endoplasmic reticulum; LATS, large tumor suppressor; MST, mammalian sterile 20-like kinase; VCP, Valosin-containing protein; YAP, Yes-associated protein

Perspective to: Kim et al. Deubiquitinase YOD1 potentiates YAP/ TAZ activities through enhancing ITCH stability. Proc Natl Acad Sci USA. 2017 May 2; 114(18):4691-4696. doi: 10.1073/pnas.1620306114. Epub 2017 Apr 17. a kinase cascade consisting of MST1/2 and LATS1/2 to control the level and nuclear localization of the transcriptional co-activators YAP/TAZ for the regulation of proliferation and apoptosis (Zhao et al. (2007) Genes Dev 21, 2747-2761). The crucial step of this pathway is the regulation of YAP/TAZ by LATS1/2 kinases. LATS1/2 phosphorylates YAP/TAZ to induce its cytoplasmic retention and subsequent proteasomal degradation. Inactivation of MST and LATS no longer phosphorylates YAP/TAZ, thus allowing the entry of YAP/TAZ to the nucleus, where it activates genes involved in cell proliferation. Recent findings reveal that regulation of the Hippo pathway is achieved at various levels via post-translational modifications, such as phosphorylation and ubiquitination.

Ubiquitin-mediated protein turnover is involved in regulating the activity of a number of Hippo pathway components. Specifically, ubiquitin-mediated LATS $1 / 2$ degradation is a major step for regulating the Hippo pathway. Convincing evidence demonstrates that the levels of LATS1/2 kinases are controlled by ITCH E3 ligase-mediated proteasomal degradation (Ho et al. (2011) Proc Natl Acad Sci USA 108, 4870-4875; Salah et al. (2011) Cancer Res 71, 2010-2020). Besides ITCH, different studies report that LATS1 and LATS2 have unique E3 ligases in different conditions. Nedd4 (neuronally expressed developmentally downregulated 4) and WWP1 E3 ligases inhibit the activity of LATS1 by targeting it for ubiquitinmediated degradation, which in turn promote tumorigenicity in various types of tumors, while SIAH1 and SIAH2 E3 ligases regulate the stability of LATS2 in response to hypoxia (Yeung et al. (2013) PLoS One 8, e61027; Salah et al. (2013) Cell Cycle 12, 3817-3823; Ma et al. (2015) Nat Cell Biol 17, 95-103).

The importance of ubiquitination in the regulation of Hippo pathway prompted us to explore the potential roles of deubiquitinases (DUBs) as regulators of the Hippo pathway. In contrast with E3 ligase, DUBs remove the ubiquitin chain from the substrate, thereby rescuing proteins from degradation. Using an unbiased DUB siRNA screen for YAP/TAZ activity, the deubiquitinase YOD1 was identified as a positive regulator of YAP/TAZ activity. YOD1 facilitates the protein quality control by VCP/p97 at the endoplasmic reticulum (ER) through the ER-associated protein degradation pathway (Ernst et al. (2009) Cell 36, 28-38). However, the function of YOD1 in regulating normal cytosolic proteins remains unclear. 
In the current study, we report that the deubiquitinase YOD1 acts as a positive regulator of YAP/TAZ in controlling the organ size. The siRNA-mediated depletion of YOD1 reduced the YAP/TAZ reporter activity and the expression of its target genes, including ANKRD1, CTGF, CYR61 and INHBA. Mechanistically, YOD1 deubiquitinates ITCH, an E3 ligase of LATS, and enhances the stability of ITCH, which leads to reduced levels of LATS and a subsequent increase in the YAP/TAZ level. The level of YOD1 is altered in a cell-densitydependent manner. Cell density is a well-known intrinsic factor that activates the Hippo pathway via enhancement of both the level and kinase activity of LATS. However, it is still unclear how cell density regulates the cellular abundance of LATS kinases. Our study showed that the increase of miR-21 levels at a high cell density is crucial for the down regulation of YOD1 and the accompanying destabilization of ITCH, which consequently increases the level of LATS. The high cell density-miR-21-YOD1-ITCH-LATS signaling cascade is parallel to a previously known high cell density-MST1/2-LATS signaling cascade. In addition to miR-21, other miRNAs such as miR-15a and miR-29b are also highly expressed in high cell density, but do not reduce YOD1 levels. Furthermore, miR-125a and miR-30b containing YOD1 target sequence markedly reduced the levels of YOD1, but the expression was not increased in high cell density. Conclusively, our data suggest that miR-21 is the only miRNA that responds to cell density and regulates the Hippo pathway by blocking the translation of YOD1.

Using a transgenic mouse model, we confirmed that the Dox-inducible expression of YOD1 in the liver enhanced the proliferation of hepatocytes, and led to hepatomegaly in a YAP/TAZ-activity-dependent manner. Also, the enlarged livers reverted to almost their normal size after stopping Dox administration, suggesting that YOD1 is sufficient to induce liver overgrowth and the overgrowth is reversible. Furthermore, analysis of clinical samples showed a strong positive correlation between high levels of YOD1 expression with YAP in liver cancer patients, indicating that YOD1 works as an oncogene,

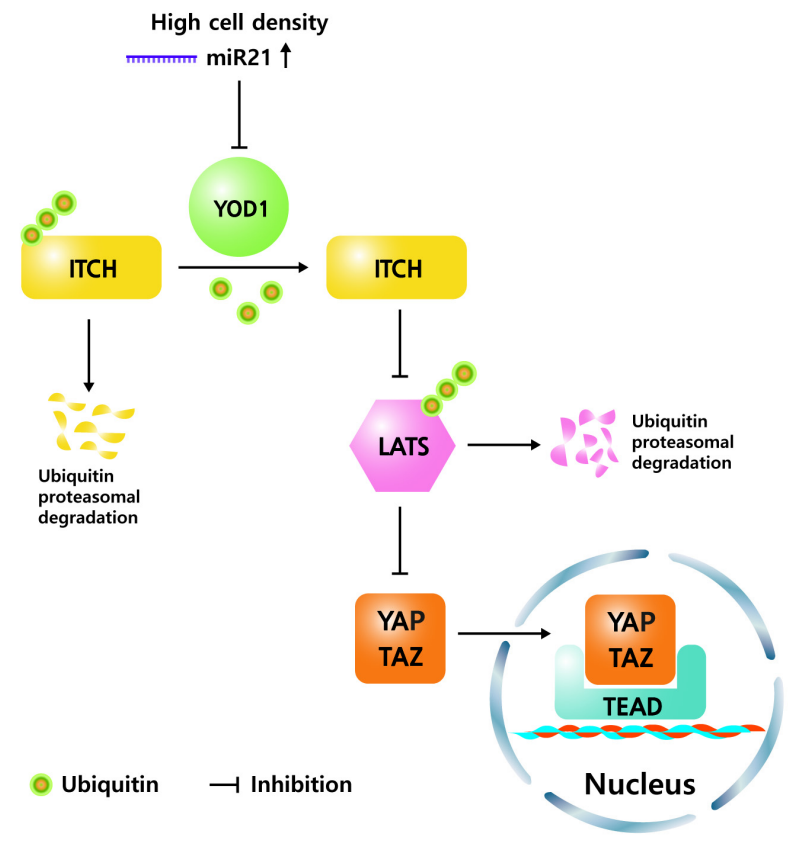

Fig. 1. Schematic model. The YOD1 maintains low levels of LATS through deubiquitination of $\mathrm{ITCH}$, which allows for high expression levels of YAP/TAZ target genes. Increase of miR21 in high cell density reduces the level of YOD1, resulting in reduction of YAP/TAZ level.

at least in liver tissue. Overall, our results uncovered YOD1 as a mediator linking the physiological signals with HippoYAP/TAZ signaling, and our studies provide a potential therapeutic target to treat liver cancer (Fig. 1).

\section{ACKNOWLEDGEMENTS}

This work was supported by the National Research Foundation of Korea (Grant NRF-2016R1E1A1A01943544) to E.-H.J. 